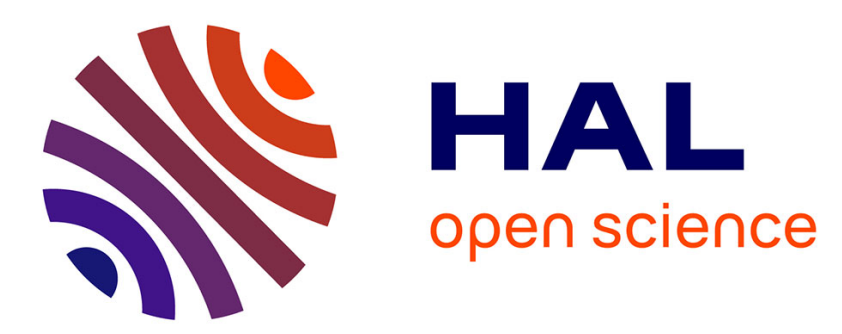

\title{
Diffusion d'un label RSE au sein d'un cluster de PME
}

Elise Bonneveux, Arnaud Gautier

\section{To cite this version:}

Elise Bonneveux, Arnaud Gautier. Diffusion d'un label RSE au sein d'un cluster de PME. Revue Internationale PME, 2019, 32 (2), pp.27. 10.7202/1062123ar . hal-02434867

\section{HAL Id: hal-02434867 https://hal.science/hal-02434867}

Submitted on 8 Apr 2020

HAL is a multi-disciplinary open access archive for the deposit and dissemination of scientific research documents, whether they are published or not. The documents may come from teaching and research institutions in France or abroad, or from public or private research centers.
L'archive ouverte pluridisciplinaire HAL, est destinée au dépôt et à la diffusion de documents scientifiques de niveau recherche, publiés ou non, émanant des établissements d'enseignement et de recherche français ou étrangers, des laboratoires publics ou privés. 


\title{
Diffusion d'un label RSE au sein d'un cluster de PME. Une analyse réticulaire fondée sur l'ANT
}

\begin{abstract}
Élise BONNEVEUX
Élise Bonneveux est maître de conférences à l'IAE de l'Université de Tours. Elle est responsable du master 2 management des PME et entrepreneuriat en formation initiale et alternance. Affiliée au laboratoire VALLOREM, ses travaux de recherche portent sur la RSE et les stratégies de réseau des petites et moyennes entreprises.

VALLOREM (EA 6296), IAE

Université de Tours

50, avenue Jean-Portalis

BP 0607

37206 TOURS CEDEX 03, France

elise.bonneveux@univ-tours.fr
\end{abstract}

Arnaud GAUTIER

Arnaud Gautier est maître de conférences à l'Université Côte d'Azur et membre du laboratoire GREDEG. Ses travaux sont tournés vers la RSE et la création de valeur partagée dans le contexte des PME et des démarches interorganisationnelles.

\author{
CNRS-GREDEG \\ Université Côte d'Azur \\ Bâtiment B \\ 250, rue Albert-Einstein \\ 06560 VALBONNE, France \\ arnaud.gautier@gredeg.cnrs.fr
}

\section{RÉSUMÉ}

La valorisation d'actions RSE mises en place par les organisations est devenue ces dix dernières années un enjeu mondial majeur. En atteste le nombre croissant de dispositifs de certification proposés aux entreprises privées comme publiques (ISO 26000, SD 21000...). Cependant, force est de constater que très souvent ces dispositifs ne sont pas adaptés au contexte de la PME, alors qu'elles représentent l'essentiel des organisations privées. Tout l'enjeu réside alors dans la compréhension d'une part, des objectifs poursuivis par les concepteurs d'un dispositif de labellisation responsable et d'autre part des besoins des organisations qui participent à un processus de labellisation pour accompagner les PME vers une performance durable. Nous nous sommes proposés, dans cette recherche, d'étudier la diffusion d'un label d'entreprise responsable (ENR) dans un réseau de clusters de PME du secteur du numérique, en mobilisant la théorie de la traduction ou théorie de l'acteur-réseau (ANT). La méthode mobilisée permet de suivre l'évolution du label ENR «en train de se développer » et d'en comprendre le succès relatif à travers l'analyse des relations entre les acteurs. Les résultats de cette étude qualitative conduisent tout d'abord à distinguer trois étapes majeures dans la diffusion de la labellisation, favorisant l'allongement $d u$ réseau et la pérennisation de l'innovation. Puis, une typologie d'engagement en faveur du label est proposée selon le degré d'intéressement et d'enrôlement des PME au sein du réseau.

\section{MOTS-CLÉS}

Théorie de l'acteur-réseau, Label, PME, Innovation, Cluster 


\title{
Dissemination of a CSR label within a cluster of SMEs. A network analysis based on the ANT theory
}

\begin{abstract}
Over the last ten years, promotion of corporate CSR actions has become a major global issue as the number of certification schemes (ISO 26000, SD 21000...) proposed by private and public organisations has increased year by year. However, we cannot but notice that these certification schemes are very often inadapted to the specific needs of SMEs, even though they account for the bulk of private organisations. What is at issue here is to understand the objectives of CSR certification schemes' designers but also the needs of the organisations that take part in labelling process, in order to accompany SMEs towards global performance. This paper investigates the dissemination of a CSR label for SMEs (ENR) within a digital media network of clusters, by mobilizing the actor-network theory. The mobilised theoretical approach allows to analyse how the label evolves and develops but also to understand the relative success of the label in so far as its examines the relationship between the actors. The main conclusions lead first to distinguish three different stages in the diffusion of the CSR labelling, fostering the development of the network but also the sustainability. Then, the authors propose a classification of engagement in favor of the CSR labelling, depending on interest and enrolment mechanisms identified within the network.
\end{abstract}

KEYWORDS

Actor-network theory. Labelling, SME, Innovation, Cluster

\section{Difusión de una marca RSE dentro de un Clúster de PyME. Un análisis reticular basado en la teoria ANT}

\begin{abstract}
RESUMEN
La valoración de las acciones RSE llevadas a cabo por las organizaciones, ha resultado ser estos últimos diez años una gran apuesta a nivel mundial. Testigos son el creciente número de mecanismos de certificación propuestos tanto para la empresa pública como privada (ISO 2600, SD 2100...). Se debe constatar que por lo general estas certificaciones no están adaptadas al contexto de la PyME, las cuales representan la gran mayoría de las organizaciones privadas. El desafío reside por lo tanto en la comprensión por una parte, de los objetivos perseguidos por los diseñadores de un dispositivo de certificación responsable, y por otra parte de las necesidades de las organizaciones que participan en un proceso de certificación para acompañar las PyME hacia una mejora continua. Este artículo estudia la difusión de la etiqueta de empresa responsable (ENR) en una red de clusters de PyME del sector digital, movilizando la teoría de la traducción o teoría del actor-red (ANT). El método desarrollado permite seguir la evolución de la etiqueta ENR "desarrollándose" y entendiendo el éxito relativo a través del análisis de las relaciones entre los actores. La primera parte expone los conceptos clave, precisa en qué la etiqueta de RSE puede ser considerada como una innovación y presenta en definitiva el acercamiento teórico desarrollado. Detallaremos a continuación el contexto estudiado y la metodología puesta en marcha antes de presentar y argumentar los resultados. Las conclusiones principales del estudio conducen en primer lugar a la comprensión de tres etapas principales en la difusión del etiquetado, favoreciendo el alargamiento de la red y la continuidad de la innovación. Por lo tanto, se propone una tipología de compromiso por la etiqueta según el grado de interés y participación de las PyME en el seno de la red.
\end{abstract}

Palabras Clave

Actor-red teoria, Marca, PyME, Innovación, Cluster 


\section{INTRODUCTION}

De plus en plus de champs organisationnels se voient traversés par la question de la responsabilité sociétale des entreprises (RSE). Ce phénomène se concrétise par le développement et la diffusion de dispositifs de gestion, centrés sur la RSE (Capron et Quairel-Lanoizelée, 2010). Moisdon (1997, p. 10) définit les dispositifs de gestion comme les «types d'arrangements des hommes, des objets, des règles, et des outils [qui] paraissent opportuns à un instant donné ». Parmi ces dispositifs, certains concepteurs optent pour la mise en œuvre de labels responsables. Tout l'enjeu théorique et managérial de l'intégration de ce type de dispositif est de comprendre comment des acteurs en arrivent à développer ces outils de gestion et les implications inhérentes à ce choix, et comment se comportent les organisations qui se trouvent confrontées à un processus de labellisation RSE. Parmi ces organisations, les PME présentent un double intérêt. D’une part, ce type d'entreprises représentent l'essentiel des organisations privées (Wymenga, Spanikova, Barker, Konings et Canton, 2012) et leurs démarches RSE se caractérisent par plusieurs facteurs : la place et le rôle joué par le dirigeant de l'entreprise (Courrent, 2012), l'implication des collaborateurs (Jenkins, 2006) et les pressions qu'elles subissent de la part des grandes entreprises (Quairel, 2006). D’autre part, ces organisations disposent statistiquement de moins d'outils RSE que des entreprises de taille plus importante (Perrini et Tencati, 2006). À l'instar de Pasquero (2004), nous associons la RSE à un paradigme novateur et réformateur, car elle incarne la volonté de diffuser un nouveau modèle de développement économique soutenable pour la planète. C'est pour cette raison qu'elle permet d'appréhender la société d'une nouvelle manière. Dans un tel contexte, les labels responsables représentent une innovation sociale dans le sens où ils participent à la production d'une valeur collective et partagée par les organisations et de ce fait tendent à transformer la société (CE, 2013 ; Cajaiba-Santana, 2014 ; Van der Yeught et Bon, 2016). Il est alors possible de mobiliser la théorie de la traduction ou théorie de l'acteur-réseau (ANT) d'Akrich, Callon et Latour $(1988,2006)$, qui consiste à mettre en avant la dimension sociale de l'innovation et à identifier les différents acteurs du réseau et leur rôle respectif dans l'élaboration de l'innovation.

De nombreux domaines de gestion, tels que l'innovation (Callon, 1986 ; Latour, 1992), le changement (Akrich, Callon et Latour, 2006) et le management (Amblard, Bernoux, Herreros et Livian, 1996) ont été étudiés à la lumière des principaux concepts de cette théorie. Citons plus récemment les travaux de thèse de Lemaire (2013) qui étudie l'implémentation d'outils de gestion dans le secteur médico-social, ceux de Bou Saba (2011) sur l'introduction d'un dispositif d'intelligence collective au sein d'un groupement de coopératives ou encore les recherches de Tanguy (2011) qui examine le processus d'externalisation d'une activité d'une organisation.

De manière générale, ce cadre théorique permet d'analyser les situations, les actants ainsi que les interactions au travers desquels les innovations sociotechniques apparaissent, se stabilisent et deviennent des faits établis qui ne sont plus mis en doute. Dans les différentes phases de conception de l'innovation, la médiation entre les futurs usagers et les concepteurs modèle l'outil dans un processus itératif de coconstruction. L'innovation est alors appréhendée comme un processus en boucle, qui se cristallise dans la collaboration des acteurs sociaux mobilisés par et dans l'élaboration même de l'objet. Selon Akrich, Callon et Latour (1988), l'innovation n'est pas un processus linéaire, mais bien une dynamique durant 
laquelle l'innovation ne cesse d'être modifiée jusqu'à la convergence des intérêts des différents acteurs et des possibilités techniques présentes. Dans cette perspective, la question de recherche de ce travail est la suivante : comment se diffuse un label RSE au sein d'un réseau de clusters de PME?

Mobilisant la théorie de l'acteur-réseau comme cadre intégrateur, la diffusion du label Entreprise Numérique Responsable mis en place par le réseau des clusters du numérique France IT est dans un premier temps étudié au niveau organisationnel pour comprendre comment l'innovation se développe au sein du réseau. Les tentatives des acteurs d'introduire, à un niveau collectif, le label ENR au sein de France IT sont examinées selon les différentes issues possibles : le succès, le détournement ou l'abandon complet du dispositif de labellisation responsable. Cette approche théorique permettant également l'analyse des jeux de pouvoir entre acteurs, nous proposons dans un second temps, une analyse complémentaire en décryptant les profils d'engagement des dirigeants de PME en faveur du label ENR. L'analyse selon deux niveaux, organisationnel en étudiant le réseau, et individuel en dressant le profil d'engagement responsable des dirigeants de PME, constitue la contribution théorique de cet article.

\section{LE LABEL RSE, UN OUTIL INNOVANT POUR LES PME}

Dans un premier temps, en nous appuyant notamment sur les travaux de RobertDemontrond et Joyeau (2007) relatifs aux labels responsables, nous soulignons l'importance et l'intérêt d'étudier les dispositifs de labellisation comme vecteurs de la RSE pour les PME. Nous nous intéressons ensuite à la diffusion de la RSE dans le contexte particulier d'un réseau de clusters de PME en mobilisant la littérature relative aux réseaux territorialisés et à la responsabilité sociétale des PME.

\subsection{Les dispositifs de labellisation comme vecteurs de la RSE}

Depuis le rapport Brundtland (1987), la responsabilité sociétale des entreprises (RSE) tend à s'institutionnaliser, au travers de plusieurs sommets (Rio, 1992 ; Johannesburg, 2002 ; COP21, 2015), de changements de législation (la loi NRE de 2003 en France par exemple), ainsi que par la création de dispositifs de gestion (Capron et Quairel-Lanoizelée, 2010). Il s'agit de labels, de normes ou de certifications RSE, citons entre autres les normes SA8000, OHSAS18001, AA1000, ISO14001 ou encore les programmes d'autorégulations WRAP, SAI, FLA, ETI, FWF et WRC (O'Rourke, 2006). Ce processus d'institutionnalisation se remarque ainsi dans différents champs organisationnels, débouchant sur la création de dispositifs de labellisation de multiples sortes et sources d'innovation pour les organisations.

La multiplication de ces dispositifs invite à établir un préalable sémantique relatif aux labels en général, et aux labels de RSE en particulier. Initialement, la création de labels s'inscrit dans une démarche commerciale visant à atténuer l'asymétrie informationnelle, dont souffrent les partenaires des organisations, et en particulier leurs clients (Carpenter et Larceneux, 2008). Ainsi, les labels dans leur fonctionnement sont soumis aux comportements des acteurs qui 
vont orienter leurs choix en fonction de l'existence ou non de ces labels. Ce comportement est structuré par leurs personnalités, leurs croyances et leurs connaissances personnelles (McEachern et Warnaby, 2008). Le fonctionnement du label dépend aussi du marché, orienté vers les professionnels ou la grande consommation (Larceneux, 2003).

S'agissant des labels RSE, Robert-Demontrond et Joyeau (2007) posent la notion de « commerce équitable " comme un praxème. Si le label " commerce équitable » a un signifiant, c'est-à-dire qu'il est porteur d'un sens, il n'a pas de signifié figé, son acceptation est ambiguë, renvoyant tout à la fois au commerce "éthique, bio et/ou ethnique ». Aussi, il prend un sens précis pour le praticien ou l'usager seulement dans le cadre de l'action. Nous prolongeons la réflexion de Robert-Demontrond et Joyeau (2007) pour l'ensemble des labels RSE, dont la signification varie en fonction des acteurs qui les désignent (Robert-Demontrond et Joyeau, 2007). Cette difficulté tient principalement au fait que le principe de labellisation RSE est relativement nouveau et que les démarches RSE recouvrent des réalités bien différentes en fonction des organisations. Ainsi, il est possible d'appréhender les labels RSE selon différents critères. Ils peuvent avoir une approche orientée produit/service ou organisationnelle. La première catégorie a une approche marketing afin de renseigner les clients sur " provenance, quality, ethics and other related values " (McEachern, 2008, p. 405). Ces labels sont regroupés sous le terme de "Values-Based Labels" (Barham, 2002). La seconde catégorie s'intéresse à l'organisation dans son ensemble. Certains de ces labels se concentrent sur un enjeu RSE spécifique, comme les labels "Égalité et Diversité », créés par le gouvernement français. D'autres en revanche se veulent très ouverts dans leur approche, comme le label LUCIE ou le BNQ 21000. Ces dispositifs peuvent également être catégorisés en fonction de leur portée qui peut être limitée à un secteur ou plurisectorielle. Enfin, le niveau d'audit et de sanctions du dispositif peut lui aussi être variable, allant de la charte déclarative (Pacte mondial), à un programme de certification rigoureux (SD 21000). Au vu de ces éléments, le label RSE laisse une possibilité de construction et d'interprétation, tant pour les acteurs responsables de son développement que pour les utilisateurs.

Par ailleurs, la diffusion d'un dispositif de labellisation RSE implique plusieurs spécificités à la fois de par la nature du dispositif, que par son processus. S'agissant de sa nature, les dispositifs de certification RSE (qu'il s'agisse de labels, de normes, de standards) s'appuient sur la prise en compte de plusieurs enjeux. Contrairement à d'autres programmes de certifications, les enjeux des dispositifs RSE sont par nature très évolutifs, de nouveaux enjeux peuvent apparaître, de nouvelles parties prenantes se manifester (Christmann et Taylor, 2006). Aussi cela oblige les acteurs, concepteurs et usagers à faire preuve d'innovation (Devaujany, 2006). Sur le processus en lui-même, Slager, Gond et Moon (2012) montrent qu'un projet de diffusion d'un dispositif RSE passe par plusieurs séquences dans le but d'obtenir un pouvoir régulateur. Ces étapes de standardisation du cadrage, d'engagement, puis de valorisation doivent reposer sur des boucles d'innovation constante, de sorte que les enjeux et les parties prenantes soient régulièrement réinterrogés dans des phases de compromis. La réussite d'un tel projet passe par l'émergence de nouveaux acteurs qui aident à la traduction des nouvelles exigences spécifiques aux programmes RSE (Slager, Gond et Moon, 2012). 


\subsection{La diffusion de la RSE au sein d'un réseau de clusters de PME}

La diffusion d'outils de gestion, et en particulier de dispositifs de labellisation dans le contexte des PME, nous apparaît comme un terrain d'étude pertinent, et cela pour plusieurs raisons.

D’abord, les PME présentent plusieurs spécificités de gestion qui, dans une certaine mesure, les rendent à même d'intégrer des éléments nouveaux dans leurs organisations comme peuvent l'être les dispositifs de labellisation RSE. Les caractéristiques intrinsèques de la PME, au regard du concept de proximité développé par Torrès $(2003,2008)$ ou de celui de l'effet de microcosme de Mahé de Boislandelle (1996), légitiment tout autant l'intérêt porté à la diffusion d'une innovation au sein de la PME. Ainsi, la faible spécialisation et le peu de formalisme qui caractérisent la PME conduisent son dirigeant à focaliser son attention, et de ce fait son action, sur ce qui se trouve proche de lui. Cette prédisposition à la prise en compte de leurs impacts positifs comme négatifs sur leur environnement proche s'explique par la proximité que ces organisations entretiennent avec leurs parties prenantes, ainsi que par leur capacité à innover rapidement, leur permettant de trouver des solutions aux problèmes sociaux et environnementaux (Oueghlissi, 2013). La littérature montre que les PME sont en effet capables de faire preuve d'une grande flexibilité et de s'approprier des innovations, en termes notamment de produits et de procédés (Barzi, 2011 ; Ajzen, Rondeaux, Pichault et Taskin, 2016 ; Trépanier et Aka, 2017).

Ensuite, la diffusion de la RSE dans une PME ne pourrait se faire sans la conviction et l'engagement de son dirigeant. De par sa personnalité (Berger-Douce, 2008) et ses valeurs (Murillo et Lozano, 2006), il conditionne fortement l'engagement RSE de son entreprise (Courrent, 2012). Comme l'expliquent Spence et Rutherfoord (2001) à partir de leur typologie de profils de dirigeants de PME, l'engagement de ces derniers est de nature diverse et conditionne la conception de la RSE au sein de l'organisation. Nous retenons à partir de cette typologie que les dirigeants de PME peuvent en fonction du rapport RSE/ambitions financières, être dans l'évitement des coûts possibles de la RSE ; utiliser la RSE comme un moyen d'atteindre les objectifs économiques; se conformer aux règles relatives à la RSE comme un moyen d'assurer la survie de l'organisation ; être avant tout mus par des considérations sociétales et environnementales.

Enfin, les PME subissent l'influence d'acteurs externes (Perrini, 2006), qui prend corps dans les attentes de leurs parties prenantes, et notamment des grands groupes qui invitent les PME à se positionner sur leurs pratiques RSE. Selon Lapointe et Gendron (2005), la RSE impacte désormais toutes les composantes de la chaîne de valeur des grandes entreprises et se répercute également sur les fournisseurs et sous-traitants. Les PME sont également touchées par ce phénomène et doivent prendre en compte ces changements, si elles souhaitent rester partenaires des grands groupes. Cette pression de l'environnement sur les PME se traduit notamment par l'intégration d'outils spécifiques, afin de répondre aux attentes des donneurs d'ordres, selon la vision messianique décrite par Quairel (2006). Selon cette approche, l'institutionnalisation de la RSE dans les secteurs économiques passe par une volonté de changement des grandes entreprises.

L'intégration des outils RSE dans le contexte des PME répond à plusieurs enjeux. En effet, les PME utilisent statistiquement moins d'outils RSE que des entreprises de taille plus 
importante, la plupart des outils existants n'étant pas adaptés aux PME (Perrini et Tencati, 2006). Ainsi, toute innovation relative à la RSE et spécifique au contexte des PME facilitera la mise en place d'actions responsables (Bonneveux, Calmé et Soparnot, 2011 ; Bonneveux et Calmé, 2015). De plus, s'il s'avère intéressant d'étudier les PME lors du déploiement de dispositifs RSE innovants, il est à supposer que ceci est d'autant plus pertinent quand il s'agit d'organisations encastrées dans des clusters. En effet, les clusters sont des réseaux territoriaux propices à l'innovation et en interaction avec les enjeux sociétaux. Le cluster se présente comme "le regroupement géographique d'organisations qui, en entretenant des relations étroites entre elles, notamment dans des activités de R\&D, impactent positivement le niveau d'innovation et le développement économique local » (Chalaye et Massard, 2009, p. 153). Ces deux dimensions de l'innovation et du territoire se retrouvent dans les travaux de Depret et Hamdouch (2010, p. 103) pour lesquels le cluster est un système d'innovation " enraciné » et " encastré » dans un territoire délimité géographiquement et équipé de différentes ressources. Ainsi, les clusters composés de PME semblent se présenter comme un terrain favorable à la diffusion d'innovations managériales (Gautier, Badea et Berger-Douce, 2017). Selon cette approche, de nombreux travaux se concentrent sur le cas des clusters du secteur numérique et s'interrogent sur leur capacité à faciliter l'action collective entre les organisations, l'open-innovation (Skog, 2016) ou encore le transfert de connaissances (Nwagwu et Ibeku, 2016), mais peu se concentrent sur la question de la RSE. Les travaux de Porter et Kramer (2011) montrent, à ce titre, la pertinence des clusters comme des écosystèmes d'innovation et le lieu d'interactions privilégiées entre les entreprises et la société, et proposent une intégration stratégique de la RSE autour des clusters par la création de valeur partagée. Au travers de ce concept, les auteurs invitent les entreprises à repenser leurs produits/services et marchés, à redéfinir leur chaîne de valeur, et finalement à s'intégrer dans un cluster local. L'encastrement territorial d'organisations en quête d'innovations partagées invite à mobiliser l'ANT pour analyser la diffusion d'une innovation au sein d'un réseau de clusters de PME.

\section{LA THÉORIE DE LACTEUR-RÉSEAU (ANT) COMME CADRE D'ANALYSE DE LA DIFFUSION D'UN DISPOSITIF DE LABELLISATION RSE}

La théorie de l'acteur-réseau (Akrich, Callon et Latour, 1988 ; 2006) apporte des clés de lecture pertinentes pour analyser une innovation managériale de RSE comme pratique de traduction des acteurs membres d'un réseau. Selon cette approche qui s'intéresse aux formes d'action organisée et aux mécanismes d'élaboration des faits sociaux (Amblard et al., 1996), ce ne sont plus les seules stratégies mises en œuvre par les utilisateurs qui façonnent l'évolution de l'innovation (modèle du déterminisme social). Au contraire, concepteurs et utilisateurs contribuent successivement à la construction sociale de l'innovation, dans une sorte d'interaction permanente. L'ANT est alors considérée comme un cadre intégrateur, permettant d'appréhender les logiques d'actions des différents acteurs constituant un réseau. Selon Callon et Latour (1991, p. 20), un acteur est «n’importe quel élément qui cherche à courber l'espace autour de lui, à rendre d'autres éléments dépendants de lui, à traduire les volontés dans le langage de la sienne propre ». Il est communément admis que la notion "d'acteur» 
soit définie selon sa dimension humaine puisque c'est une personne qui agit. Callon et Latour vont au-delà de cette représentation en intégrant dans la définition tout ce qui est en mesure d'agir, d'impacter positivement ou négativement une action. Ils reprennent la notion « d'actant » développée par Greimas (1986) dans ses travaux de sémantique. Les actants constituent ainsi l'ensemble des acteurs humains et non humains (par exemple le réseau, le dispositif) qui occupent une place égale dans le processus de coconstruction de l'innovation (Bloor, 1976 ; Latour et Lemonnier, 1994).

Cette théorie nous conduit à analyser les relations de pouvoir, la recherche d'accords impliquant la confrontation d'intérêts incompatibles, en proposant d'observer et de décrire les interactions entre « actants». Ainsi, en ajoutant au pouvoir des acteurs humains celui des nonhumains, le réseau gagne en substance (Callon, 2006). Dans ces conditions, pour décrire l'élaboration d'une innovation, il faut étudier le réseau au travers lequel elle se développe. En effet, selon Akrich, Callon et Latour (1988, p. 17), "l'innovation, c'est l'art d'intéresser un nombre croissant d'alliés qui vous rendent de plus en plus fort ».

Cette hypothèse constitue un prolongement des apports de l'ANT selon lesquels l'appropriation d'une innovation nécessite la mise en place de processus "d'intéressement » qui s'amplifient avec le temps. L'implication des individus dans la construction d'une innovation apparaît comme une des conséquences possibles des mécanismes d'intéressement et d'enrôlement que certains individus sont parvenus à pérenniser. Le modèle " d'intéressement » permet de souligner l'existence de relations qui unissent les acteurs de l'innovation managériale et met en exergue les intérêts individuels et collectifs dans la diffusion du dispositif de RSE.

Ainsi, l'innovation va connaître plusieurs adaptations qui résultent le plus souvent d'un accomplissement collectif, résultat d'un intéressement de plus en plus vaste. Avec l'ANT, il est possible de traduire le processus qui permet au fait de se construire. Amblard et al. (1996, p. 135) donnent une définition de la notion de traduction : " Dans le langage courant, traduire renvoie à une opération qui consiste à transformer un énoncé intelligible en un autre énoncé intelligible pour rendre possible la compréhension de l'énoncé initial par un tiers. La traduction est réussie si elle n’a pas engendré un détournement de sens. »

Autrement dit, la traduction est la conséquence d'une négociation entre différentes parties. Le traducteur est l'acteur qui aide et nourrit le lien qui relie les parties prenantes constituant le réseau et c'est à travers lui que s'opère la problématisation. Selon Nobre et Zawadski (2015), le rôle de traduction doit être confié à un acteur qui bénéficie de la légitimité des autres membres du réseau. Cette légitimité acquise, le traducteur peut alors procéder à l'alignement des traductions de chacun des protagonistes. Le processus de traduction peut être analysé selon trois étapes (Callon, Lascoumes et Barthe, 2001) : l'analyse du contexte et la problématisation de la situation (1), l'animation d'un collectif pour proposer un point de passage obligé (2), l’allongement et l'irréversibilité de l'innovation (3).

\section{Étape 1 : analyse du contexte et problématisation de la situation}

L'analyse du contexte revient à une présentation des « actants » participant au processus, de leurs enjeux respectifs et de leurs intérêts, communs et convergents. L'ensemble des entités présent dans l'élaboration du projet doit donner lieu à une analyse. La problématisation est le processus par lequel les acteurs « acceptent » la manière, dont les groupes de réflexion 
définissent le problème. Le traducteur repère ce qui unit et ce qui sépare les acteurs, puis il formule le projet d'innovation sous la forme d'une question qui permet leur convergence.

\section{Étape 2 : animation d'un collectif pour proposer un point de passage obligé}

Il appartient alors au groupe de réflexion du réseau d’intéresser les acteurs précédemment identifiés, c'est-à-dire de les mobiliser de façon durable. L'intéressement est l'ensemble des actions par lesquelles le groupe de réflexion tente de stabiliser l'identité des acteurs autour de l'innovation. Le collectif sélectionne et met en forme ces points de vue opposés, différents, éparpillés en faisant le choix de certains raisonnements logiques, de certaines présentations statistiques, etc. Ce travail a pour objet la définition de ce que Akrich, Callon et Latour (1988) appellent un «point de passage obligé ».

\section{Étape 3 : allongement du réseau et irréversibilité de l'innovation}

Peu à peu le réseau se solidifie. Les jeux de pouvoir autour des porte-parole, des problèmes à résoudre, des intermédiaires s'estompent et les relations se cristallisent. Les actions et choix admis par le collectif deviennent des boîtes noires qui ne font plus l'objet d'aucune discussion. Allonger le réseau, c'est intéresser de nouvelles personnes à l'innovation en cours et trouver de nouveaux moyens pour les unir autour du projet de changement. Il s'agit à la fois de multiplier les entités (structures, accords, moyens) qui composent le réseau et d'intéresser des acteurs occupant une position stratégique (leaders d'opinion, responsable de l'affectation de moyens, etc.). Plus le réseau est développé et plus l'innovation revêt un caractère irréversible.

\section{PRÉSENTATION DE LA MÉTHODOLOGIE ET DU TERRAIN DE LÉTUDE}

Ce travail de recherche s'appuie sur l'étude d'un cas de déploiement d'un label RSE au sein du secteur numérique français. Nous présentons d'abord l'environnement de ce déploiement en apportant des éléments de contexte définissant le secteur du numérique, ainsi que l'association professionnelle France IT. Puis, nous exposons les principes du label Entreprise Numérique Responsable ainsi que le processus de labellisation. Enfin, nous présentons les éléments de méthodes adoptés de collecte, de traitement et d'analyse des données.

\subsection{L'association de clusters du secteur numérique : France IT}

Depuis le début des années deux-mille, le secteur du numérique est en pleine croissance. Le développement des sites marchands et des applications, fait des entreprises du numérique un acteur central pour l'ensemble des organisations privées comme publiques. Ainsi, le secteur représente environ $4 \%$ de la croissance dans les pays européens. En France, le chiffre d'affaires était de 61,4 milliards d'euros en 2014, pour atteindre 71,3 milliards d'euros en 2015. Sur la même période, le nombre de sites marchands en France a augmenté de plus de $15 \%$ (Observatoire du numérique, 2015). Cette croissance constante et les nouveaux usages posent un certain nombre d'interrogations relatives au respect de la vie privée des 
utilisateurs, comme des collaborateurs (Sama et Shoaf, 2002 ; Koehn, 2003), à la sécurité des données (Koehn, 2003), à la propriété intellectuelle (Sama et Shoaf, 2002 ; De George, 2003), à la transparence des informations (Sama et Shoaf, 2002 ; Koehn, 2003), aux conditions de travail dues aux nouvelles formes de métiers (Fieseler, Bucher et Hoffman, 2017), aux enjeux relatifs à la parité (Panteli, Stack et Ramsay, 1999) ou encore à la question de l'éthique dans les pratiques des développeurs (Shilton et Greene, 2017).

L'association France IT, créée en 2009, regroupe en 2017 treize clusters du secteur numérique à travers la France. Chacun de ces clusters compte environ une centaine d'entreprises, pour l'essentiel des PME, voire des TPE. France IT a deux objectifs : le premier est d'aider les clusters dans l'accompagnement des entreprises et dans la promotion d'actions communes entre les clusters. Le second est d'accompagner les entreprises membres dans leurs objectifs de performance et faciliter les connexions entre entreprises du secteur.

\subsection{Le dispositif de labellisation Entreprise Numérique Responsable}

C'est dans ce cadre que le dispositif de labellisation ENR a été développé à partir de 2010 et diffusé depuis 2011. Les objectifs du label ENR sont de participer à la structuration du secteur qui, comparativement à d'autres, est encore jeune (Panteli, Stack et Ramsay, 1999) et de le faire notamment autour de pratiques responsables. Comme nous l'avons souligné, le secteur soulève plusieurs enjeux et souffre de plusieurs a priori, concernant notamment l'éthique des affaires, le manque de lisibilité de ses offres et une prise en considération de ses impacts sociaux et environnementaux limités. Ce constat se voit renforcé par l'absence de comportements éthiques normatifs pour les entreprises du numérique (Sama et Shoaf, 2002). À l'initiative du directeur de France IT, de l'actuelle présidente du cluster A et de l'ancien président de ce même cluster, le label a été créé autour de groupes de travail réunissant des entreprises membres des clusters afin de délimiter les questions relatives à la RSE. Le processus de labellisation se décompose en quatre phases. Entre février et octobre de chaque année, les entreprises ont la possibilité de monter un dossier de candidature et de le transmettre à l'organisme tiers-certificateur, le Centre international de ressources et d'innovation pour le développement durable (CIRIDD). Ce dossier de candidature s'appuie sur un référentiel de labellisation comprenant plusieurs thématiques, telles que les relations employeurs/employés et le climat social, la gestion et la protection de l'environnement, l'utilisation des ressources, l'éthique des affaires, la gestion des données et la propriété intellectuelle (1). Entre novembre et décembre, les dossiers de candidature sont instruits par le CIRIDD. Dans le même temps, une enquête auprès des entreprises clientes est menée via une plateforme web. Cette enquête porte sur l'éthique des affaires et la transparence des entreprises candidates (2). Au cours du mois de janvier, le CIRIDD présente devant le Comité d'attribution du label (CATLAB), composé de France IT et de membres de syndicats du secteur numérique, les dossiers évalués sur une note de 200, le score de 120 points étant requis pour accéder à la labellisation (3). Au mois de février, le résultat des entreprises reçues est communiqué, ces dernières ont alors la possibilité d'afficher le label sur leur support de communication (4). Chaque année, le processus de labellisation est proposé aux entreprises membres des clusters sous forme de vague de labellisation. 


\section{3. Éléments de méthode}

Cette étude s'inscrit dans une approche qualitative à visée exploratoire. Cette démarche s'explique par le fait que peu de travaux renvoient à notre question de recherche, ce qui justifie également notre stratégie d'accès au réel par le biais d’une étude de cas (Yin, 2014).

Le déploiement de cette enquête exploratoire s'est articulé autour de trois méthodes de collecte de données. Nous avons mené des entretiens de type semi-directif auprès de 40 acteurs : d'abord les concepteurs du dispositif, que nous avons interviewé à plusieurs étapes de ce processus de recherche, des dirigeants de PME labellisées, des membres des instances de gouvernance des clusters, des chargés de mission, l'organisme tiers-certificateur (CIRIDD), un représentant du comité d'attribution du label, des directeurs de clusters, et d'anciens directeurs de clusters ouvertement sceptiques vis-à-vis du dispositif de labellisation (Tableau 1). Ces entretiens ont été conduits en nous appuyant sur un guide d'entretien élaboré à partir des champs théoriques mobilisés. D'une durée moyenne d'une heure et demie, l'ensemble des entretiens a été intégralement enregistré puis retranscrit. En parallèle, nous avons procédé à des phases d'observation lors de la procédure d'instruction des dossiers de labellisation auprès de l'auditrice de l'organisme auditeur. Enfin, ces deux méthodes ont été complétées par le recueil de données secondaires auprès de l'ensemble de ces acteurs (Yin, 2014).

À l'aide du logiciel NVivo 10, nous avons procédé au codage des données selon trois étapes. Nous avons d'abord déterminé l'unité d'analyse pertinente en vue de notre analyse des données, c'est-à-dire un ensemble de phrases portant sur une thématique précise (Bernard et Ryan, 2010). La deuxième étape correspond à notre premier niveau de codage. Celui-ci avait pour objectif d'être descriptif et proche des retranscriptions et des thèmes évoqués. Enfin, la dernière étape correspond au deuxième niveau de codage, l'objectif étant de créer un arbre thématique afin de « remonter» en abstraction, de sorte que nous puissions procéder à une analyse itérative entre les données collectées et notre cadre théorique. 


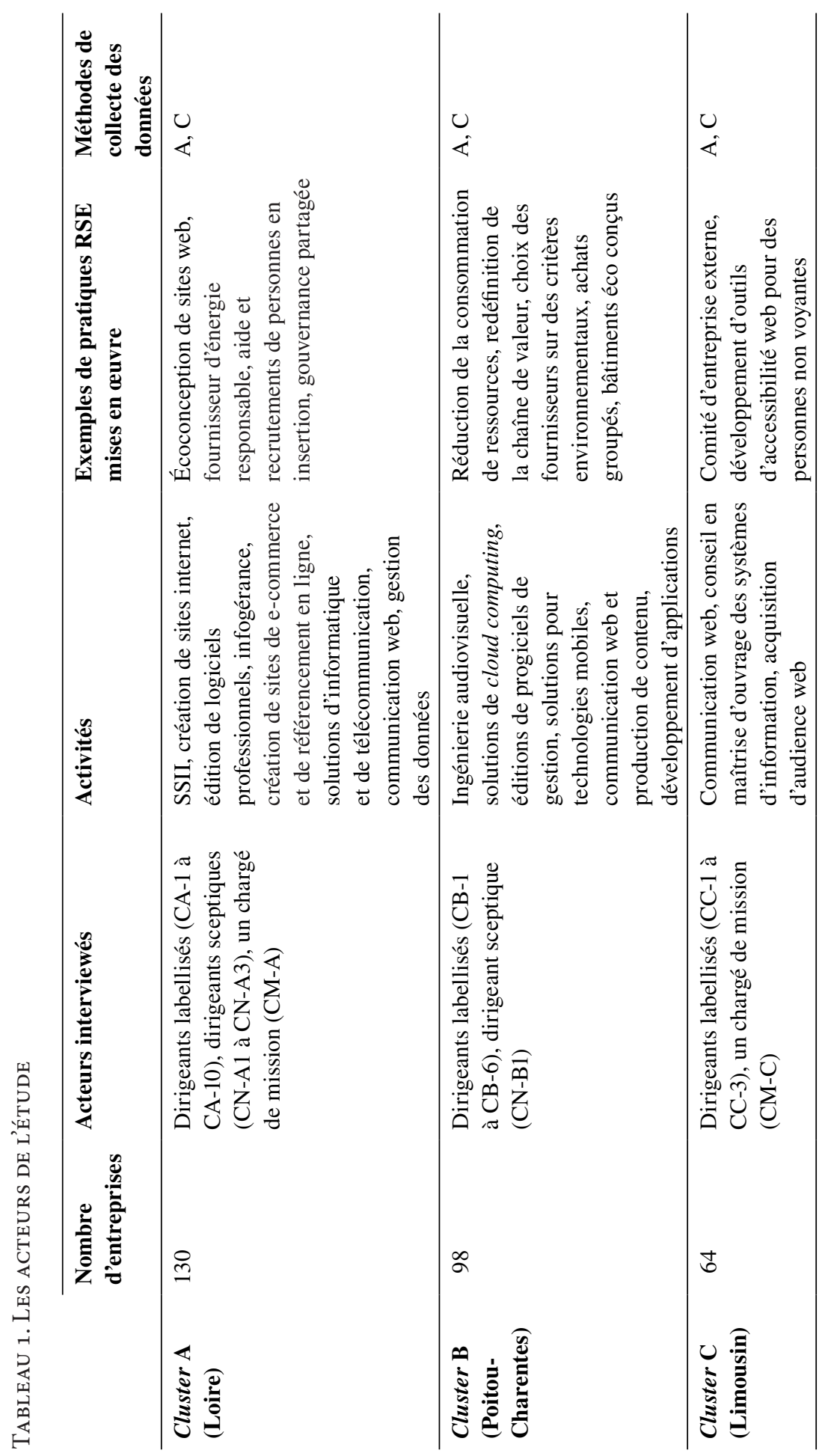




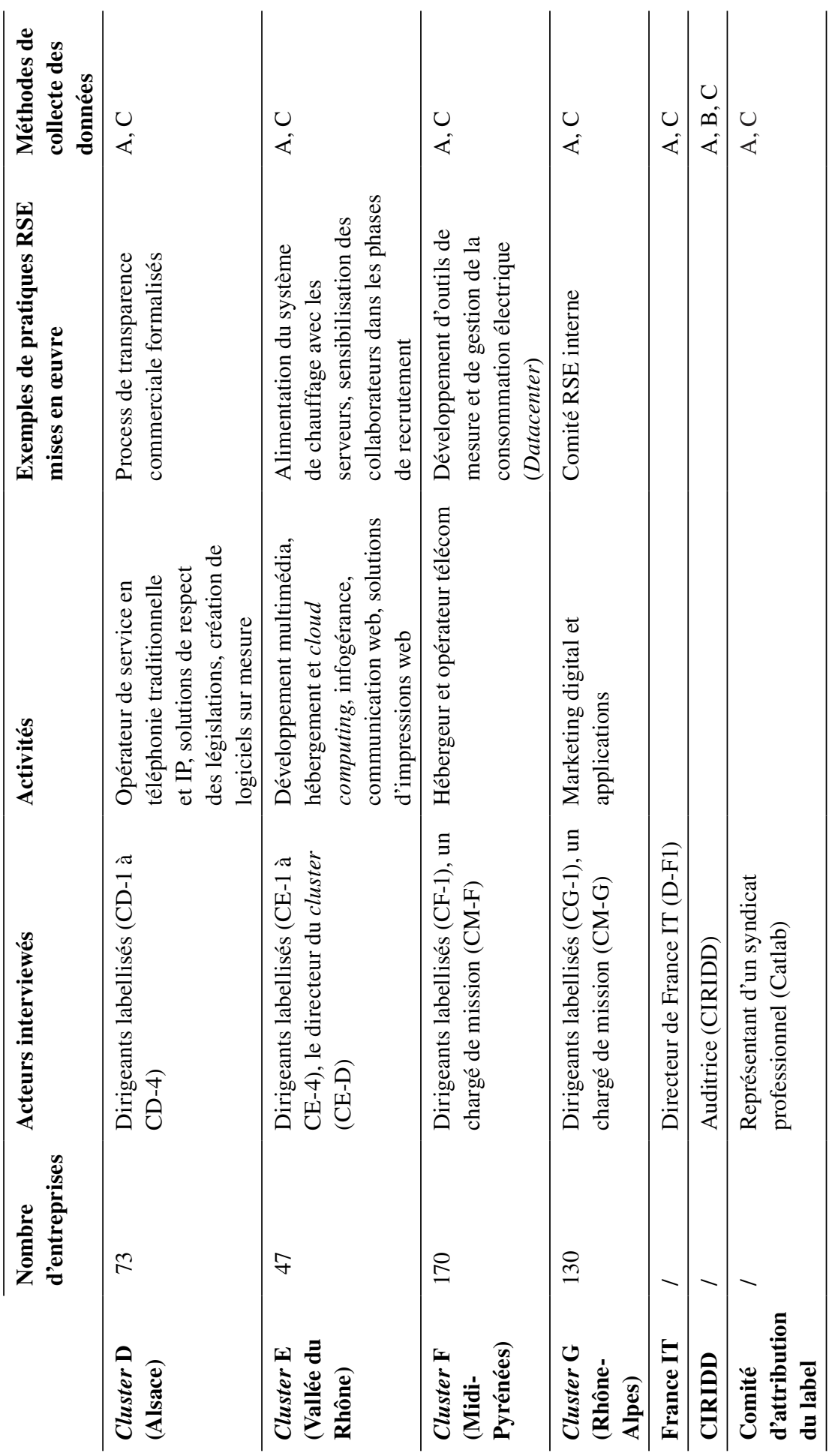




\section{PRÉSENTATION DES RÉSULTATS}

Les résultats de l'étude sont présentés en deux temps. Tout d'abord, le processus de traduction du label ENR au sein du réseau est étudié. Nous proposons ensuite une typologie d'engagement des dirigeants construite à partir des mécanismes d'intéressement et d'enrôlement identifiés au sein du réseau.

\subsection{Analyse du processus de traduction du label ENR}

Nous proposons une méthodologie d'analyse à un niveau collectif du processus de traduction du label ENR qui, d'après les travaux de Callon, Lascoumes et Barthe (2001), peut être étudiée en trois temps. Nous adaptons ce découpage à notre lecture du phénomène observé. Si pour des raisons de commodité, nous présentons chacune des étapes du processus de traduction de manière chronologique, il est à souligner qu'elles peuvent également être examinées selon un découpage analytique (Amblard et al., 1996). La figure 1 présente la constitution du label ENR ainsi que la traduction des intérêts des membres de l'association France IT, au travers du processus «d'intéressement». La partie supérieure de la figure décrit les trois étapes du processus de traduction du label ENR. Celles-ci se traduisent par la prise de responsabilités et l'enrôlement des acteurs autour du directeur de France IT et de l'actuelle présidente du cluster $\mathrm{C} 1$ et de l'ancien dans le réseau. La partie inférieure montre la mobilisation des dirigeants membres de France IT. Elle est représentée par les différentes actions proposées par l'association, favorisant l'appropriation du label ENR.

\section{Figure 1. Constitution du label ENR et mobilisation des aCteurs du RÉSEau}

\begin{tabular}{|c|c|c|c|c|c|}
\hline \multicolumn{2}{|c|}{ Constitution du label ENR } & & & & \\
\hline \multicolumn{6}{|c|}{ Acteurs au centre du réseau : } \\
\hline $\begin{array}{l}\text { Instances de } \\
\text { gouvernance de } \\
\text { clusters ( } 3 \text { acte } \\
\text { clés) }\end{array}$ & $\begin{array}{l}3 \text { acteurs clés } \\
+ \text { CIRIDD }\end{array}$ & $\begin{array}{l}3 \text { acteurs clés } \\
\text { +CIRIDD : tiers } \\
\text { certificateur } \\
+ \text { Comité } \\
\text { d'attribution du } \\
\text { label }\end{array}$ & $\begin{array}{l}\text { Désengagement } \\
\text { de } 2 \text { des acteurs } \\
\text { clés au profit de } \\
\text { France IT } \\
\text { + Nouvel } \\
\text { auditeur au } \\
\text { CIRIDD }\end{array}$ & $\begin{array}{l}\text { France IT } \\
+ \text { +CIRIDD }\end{array}$ & $\begin{array}{l}\text { France IT } \\
+ \text { CIRIDD }\end{array}$ \\
\hline \multicolumn{2}{|c|}{$\begin{array}{l}\text { Etape } 1 \text { : Analyse du contexte } \\
\text { et problématisation de la } \\
\text { situation }\end{array}$} & \multicolumn{2}{|c|}{$\begin{array}{l}\text { Etape } 2 \text { : Animation d'un } \\
\text { collectif pour proposer un point } \\
\text { de passage obligé }\end{array}$} & \multicolumn{2}{|c|}{$\begin{array}{l}\text { Etape } 3: \text { Allongement du } \\
\text { réseau et irréversibilité de } \\
\text { l'innovation }\end{array}$} \\
\hline $2009 / 2010$ & $2011 / 2012$ & 2012 & 2013 & 2014 & 2015 \\
\hline $\begin{array}{c}\text { Création de } \\
\text { France IT } \\
\text { Diagnostics du } \\
\text { secteur du } \\
\text { numerique et des } \\
\text { certifications RSE }\end{array}$ & $\begin{array}{c}\text { Groupes de } \\
\text { travail } \\
\text { Définition et } \\
\text { rédaction du } \\
\text { référentiel du } \\
\text { label ENR }\end{array}$ & $\begin{array}{l}\mathbf{1}^{\mathrm{re}} \text { vague de } \\
\text { labellisation }\end{array}$ & $\begin{array}{l}2^{\mathrm{c}} \text { vague de } \\
\text { labellisation }\end{array}$ & $\begin{array}{l}3^{e^{e}} \text { vague de } \\
\text { labellisation }\end{array}$ & $\begin{array}{l}4^{\mathrm{e}} \text { vague de } \\
\text { labellisation }\end{array}$ \\
\hline \multicolumn{6}{|c|}{ Appropriation du label ENR par lẹs actions proposées : } \\
\hline $\begin{array}{l}\text { A ce stade, les } \\
\text { entreprises ne } \\
\text { sont pas } \\
\text { encore } \\
\text { directement } \\
\text { impliquées }\end{array}$ & $\begin{array}{l}\text { Action collective } \\
\text { de sensibilisation } \\
\text { proposée aux } \\
\text { entreprises des } \\
\text { clusters } \\
\text { Accompagnement } \\
\text { par un cabinet de } \\
\text { conseils }\end{array}$ & $\begin{array}{l}\text { Accompagnement } \\
\text { par un chargé de } \\
\text { mission } \\
14 \text { entreprises } \\
\text { labellisées }\end{array}$ & $\begin{array}{l}\text { Accompagnement } \\
\text { par un chargé de } \\
\text { mission } \\
7 \text { entreprises } \\
\text { labellisées }\end{array}$ & \multirow[t]{2}{*}{$\begin{array}{l}\text { Légitimation } \\
\text { des clusters } \\
9 \text { entreprises } \\
\text { labellisées }\end{array}$} & $\begin{array}{l}\text { Stagnation du } \\
\text { nombre } \\
\text { d'entreprises } \\
\text { labellisées } \\
\text { Action de } \\
\text { communication } \\
\text { proposée }\end{array}$ \\
\hline \multicolumn{4}{|c|}{ Mobilisation des acteurs du réseau } & & \\
\hline
\end{tabular}


Au cours de ces trois étapes, nous soulignons maintenant le rôle de traducteur assuré par les concepteurs et parties prenantes au dispositif de labellisation, et comment ceux-ci s'appuient sur le réseau de clusters existant.

Étape 1 : analyse du contexte et problématisation de la situation

Trois acteurs sont à l'initiative du dispositif de labellisation, à savoir le directeur de l'association France IT (D-F1), l'actuelle présidente du cluster A (CA-1) et l'ancien président de ce même cluster (CA-2). Ces trois responsables peuvent être désignés comme les acteurs qui impulsent le changement. Callon et Latour (1991) les appellent les primum movens. En effet, à partir de 2009, ces acteurs vont faire le diagnostic du secteur du numérique en France, mettant ainsi en évidence les limites, dont souffre ce secteur. Parmi celles-ci, D-F1, CA-1 et CA-2 soulignent le caractère instable du secteur qui s'explique notamment par sa jeunesse.

"Le numérique, c'est un métier récent, beaucoup de jeunes entreprises, petites. » (CA-1)

Comme l'explique CA-2, le secteur du numérique a connu une période de croissance très rapide lors de la bulle informatique à la fin des années quatre-vingt-dix, début des années deux-mille qui a participé à l'instabilité du secteur, celui-ci étant en cours de structuration aujourd'hui. Toutefois, et comme le souligne le dirigeant de l'entreprise labellisée CA-3, cette instabilité sectorielle n'est pas totalement une chose ancienne, et ce ressenti persiste au sein même des organisations :

"Où en sera l'entreprise dans deux ans, on ne peut pas le savoir. Sera-t-elle rachetée par un groupe, phagocytée par un concurrent, on ne sait pas ça."

Cette jeunesse et cette instabilité d'un secteur en structuration peuvent être perçues par les parties prenantes des acteurs du numérique comme un élément négatif. Le diagnostic réalisé met en évidence cette limite et plus largement la question de la perception des prestations proposées par les entreprises du secteur.

"On est en plus dans des métiers qui sont totalement abstraits donc l'acheteur de service numérique comprend mal ce qu'il est en train d'acheter et comprend mal ce qu'on lui a livré. Donc on a très souvent des acheteurs qui sont mécontents ou qui sont inquiets. [...] " (CA-1) Pour CA-2, au-delà de l'aspect purement technique des prestations qui peuvent sembler complexes et lointaines pour les acheteurs, la bulle informatique, précédemment évoquée, a fortement contribué à ce déficit d'image :

"Avec la bulle sont apparus tous types de personnes, qui ont profité de la rareté de l'offre, pour proposer des produits ou des services extrêmement chers. Avec une qualité plus ou moins variable. Les gens ayant peu de retours, peu de visibilité sur ce qui devait être une bonne prestation, se sont empressés de prendre le plus agréable, le plus sympathique, le plus charmeur. Des fois, cela marchait très bien, parce que la prestation correspondait aux attentes, et les clients n'étaient pas informés de ce que l'on pouvait avoir ailleurs. Des fois, cela se passait très mal. »

Malgré cette perception souvent négative des entreprises du numérique, le secteur bénéficie des évolutions structurelles de l'ensemble des activités économiques autour des technologies de l'information et des communications, leurs services devenant ainsi incontournables. 
"Il y a quelque chose que l'on constate dans pratiquement tous les secteurs de l'informatique et de l'édition de logiciel, c'est que notre filière est en mesure d'apporter à d'autres un outil qui est conforme à leurs besoins et attentes. " (CA-1)

Il est un troisième point que le groupe de primum movens a su relever au cours de son diagnostic. Si les entreprises du numérique sont de plus en plus sollicitées, de par les changements de pratiques des organisations tous secteurs confondus, la question de la responsabilité sociétale des organisations se fait elle aussi de plus en plus présente dans les différents champs organisationnels. Sur cet aspect, le secteur du numérique connaît quelques lacunes.

"Beaucoup d'entreprises dans le réseau, quand on a commencé à diffuser, je me souviens, disaient, nous on n'est pas concernés parce qu'on n'est pas une entreprise de transport, on ne travaille pas dans les produits chimiques, nous tout est dématérialisé donc en gros on ne consomme pas. Un des grands enjeux environnementaux c'est l'IT. [...] On sait aujourd'hui que pour fabriquer nos jolis ordinateurs sont utilisés les métaux rares et que l'on appauvrit de façon dramatique certains territoires. On sait que l'on construit je ne sais pas combien de DATA Center par jour parce que notre courriel il passe trois fois aux USA, deux fois au Canada avant de revenir ici. » (D-F1)

Ce constat est partagé par les deux autres primum movens et s'étend également aux questions des pratiques sociales et de la responsabilité territoriale des organisations vis-à-vis de leurs communautés locales.

\section{Étape 2 : animation d'un collectif pour proposer un point de passage obligé}

La dynamique de la deuxième étape du processus comprend d'une part le diagnostic des dispositifs existants au sein des clusters, et d'autre part le déploiement d'une démarche d'action collective avec des dirigeants de PME autour du développement durable. En parallèle, l'association France IT est en train de se constituer, ce qui va renforcer le collectif naissant.

À partir de leur diagnostic, les primum movens déterminent une solution présentée comme le point de passage obligé aux autres acteurs. En choisissant de concevoir un dispositif de gestion, les trois responsables prennent en considération les dispositifs déjà existants et leurs limites. C'est ainsi que confrontés à la question des pratiques de leurs membres en termes de développement durable et d'éthique des affaires, plusieurs clusters du numérique ont développé et diffusé des chartes de bonnes pratiques qui s'imposent au sein du réseau comme des " actants", ces acteurs non humains qui favorisent la diffusion de l'innovation. C'est le cas notamment du cluster CA, "cette charte a été signée pendant de nombreuses années par pratiquement la moitié de nos adhérents qui s'y sont intéressés " (CA-2), le problème étant l'aspect purement déclaratif de la charte qui, de par son faible niveau d'exigence, est signée par des acteurs peu enclins à respecter leur engagement.

"Il semblait que ces bonnes pratiques n'étaient pas tellement respectées alors qu'ils étaient signataires de la charte. » (CA-1)

En plus de cet aspect, le directeur de France IT (D-F1) reconnaît le côté trop localisé et limitant des chartes "mais bon c'est du loco-local, ce n'est pas très valorisant", ainsi que le foisonnement d'initiatives de ce type au sein du secteur, rendant l'action, la portée et la lisibilité de chacune des chartes très limitées "[...] le problème avant, c'est qu'il y avait beaucoup de chartes "éparpillées" dans les différents clusters, chacun avait rédigé sa propre charte». 
France IT devient alors le traducteur, à savoir l'acteur central du processus de traduction en assurant le lien entre tous les membres du réseau. Dans la continuité de cette démarche collective, les trois primum movens ont souhaité poursuivre l'expérience en développant un projet de création de label sectoriel ayant trait à la responsabilité sociétale des entreprises du numérique. Partant de l'expérience d'actions collectives et dans le but de rallier d'autres acteurs du secteur, ils choisissent de co-créer un référentiel RSE en mobilisant cinq clusters du secteur et une trentaine d'entreprises membres. Scindées en groupe de travail, ces entreprises vont augmenter, modifier et faire évoluer le référentiel sur ce qui est, selon elles, une entreprise numérique responsable. À partir des documents issus des groupes de travail, un cabinet de conseil spécialisé dans le développement durable et la RSE va donner une cohérence globale aux différentes approches et finaliser le référentiel du label ENR.

Cette phase intervient en même temps que la création de l'association France IT. La création de ce label et la participation à des groupes de travail, est à la fois un moyen pour se rendre légitime auprès des clusters et des entreprises membres et à leur proposer une solution pertinente au regard du diagnostic établi. Nous comprenons ici la légitimité selon l'approche d'Aldrich et Fiol (1994) basée sur les dimensions cognitive et sociopolitique. En effet, les concepteurs du dispositif cherchent à développer un projet qui soit compréhensible par les parties concernées, sans que cela ne nécessite une pensée consciente (DiMaggio et Powell, 1983). Ce dispositif doit également permettre à France IT d'apparaître comme socialement acceptable et cohérent par rapport à ce qui est attendu des missions de l'association, eu égard à la dimension sociopolitique de sa légitimité. La création d'un label devient alors le point de passage obligé pour animer ce collectif d’acteurs, répartis sur tout le territoire national :

"En 2009, on a eu quelques échanges entre clusters du numérique français. Finalement, on a créé l'association France IT en 2010. Elle rassemble actuellement 14 clusters sur l'ensemble du territoire national, ce qui correspond à environ 2000 entreprises. »(D-F1)

L'avantage de France IT est de pouvoir donner à son dispositif une portée nationale contrairement aux chartes locales :

"À un moment donné, on s'est dit, on a tout de même la légitimité pour aller au-delà d'une simple charte déclarative, on va essayer de construire un label et on va prendre une dimension nationale, cela s'est trouvé au même moment que la construction de France IT, en confiant à France IT ce label. »(CA-1)

Cette légitimité se voit ainsi renforcée par les porte-parole que sont les instances de gouvernance des clusters, premiers acteurs à relayer l'existence du label, le travail du cabinet de conseil responsable de la finalisation du référentiel du label ENR ainsi que la présence du CIRIDD comme organisme tiers auditeur.

\section{Étape 3 : allongement du réseau et irréversibilité de l'innovation}

Ce travail de France IT se poursuit dans une troisième étape d'élargissement du réseau, par le biais d'un enrôlement d'acteurs, notamment institutionnels. En parallèle, nous assistons à un désengagement de deux primum movens. Malgré ce travail de France IT, cette étape est marquée par les limites que rencontre la diffusion du dispositif. 
En se présentant comme une supra-instance des clusters, et en se servant des instances de gouvernance comme d'un relai d'informations du label, France IT, en tant qu'acteur légitime du changement, élargit le réseau du dispositif en le calquant sur son réseau de membres.

"Chaque cluster a été formé au sein de France IT à accompagner les entreprises candidates, avec un document formalisé qui dit comment on peut accompagner chaque entreprise sur chaque question. " (D-F1)

Ce rôle de relai du dispositif de labellisation est intégré par les instances de gouvernance des clusters, à l'image du chargé de mission du cluster F (CF-CM) :

«Nous proposons systématiquement un accompagnement. Ne serait-ce que pour revoir la manière de présenter les choses. Parfois aussi les chefs d'entreprises ou les personnes responsables de la rédaction du dossier ne pensent pas à mettre tous les éléments. "

Ce travail de diffusion et d'enrôlement se poursuit par France IT à un niveau national. Lassociation poursuit une action de lobbyisme auprès notamment des consulaires dans le but « [...] d'imposer, proposer, le label auprès de prescripteurs, auprès des clients de nos entreprises» (D-F1) et ainsi allonger le réseau et rendre l'innovation irréversible. L'intégration d'acteurs institutionnels au processus de labellisation, notamment les syndicats professionnels qui font partie intégrante du comité d'attribution du label, donne un caractère encore plus fort à la démarche, "c'est important parce que les membres d'attribution du label sont des gens qui ont des fonctions importantes dans la filière numérique en France. Donc, on les sort comme ça de leur profession, un instant pour les faire se rejoindre sur un truc commun autour d'une table. Et puis cela montre aussi la légitimité du label " (Catlab). Ces nouveaux acteurs deviennent à leur tour porte-parole dans le processus de diffusion du label ENR.

Le caractère irréversible du dispositif se voit renforcé par la décision de deux des trois primum movens de se retirer de la supervision du projet une fois la diffusion lancée. Ce choix est expliqué par CA-1, afin de crédibiliser le dispositif en lui donnant une certaine indépendance entre concepteurs et auditeurs :

"Nous on a fait un travail de préparation de ce label, mais ensuite ne serait-ce que pour des raisons déontologiques, il a été confié uniquement à France IT."

Ce retrait de deux concepteurs et l'obligation d'indépendance donnée au label retirent à celui-ci son pouvoir de transformation :

"Nous avons fait quelques réunions après coup avec le cluster C1 pour voir l'impact du label notamment sur les relations clients, mais nous n'avons plus la main sur le label en lui-même.» (CA-2)

Cela dit, et malgré l'avantage pour les concepteurs du dispositif de s'appuyer sur un réseau de clusters déjà existant, la diffusion du dispositif de labellisation tend à s'essouffler à partir de la quatrième vague de labellisation. En effet, comme l'explique le directeur de France IT (DF-1) :

"On est dans une phase d'ascension qui est trop lente, moi je trouve, je suis plutôt impatient comme bonhomme, et pour avoir cette communication nationale, on est obligé d'avoir un seuil. » 
Au niveau du collectif, les premières étapes du processus de traduction ont bien été respectées : la problématisation, le choix du traducteur, l'animation d'un collectif et la création d'un point de passage obligé se sont opérés sans détournement de la part des acteurs du réseau. L'allongement du réseau s'est ensuite réalisé progressivement, en faisant appel à des intermédiaires, accompagnant le processus de traduction (les autres clusters territoriaux) ainsi qu'à des porte-parole (instances institutionnelles), mais l'essoufflement identifié lors de la quatrième phase et par conséquent le succès mitigé de la diffusion du label ENR au sein du réseau s'explique par un manque d'enrôlement et d'implication des dirigeants de PME dans la coconstruction du dispositif de labellisation. Cet enrôlement, qui se caractérise par une prise de responsabilité au sein du réseau, est essentiel à la bonne diffusion de l'innovation. Une analyse complémentaire, à un niveau individuel, permet d'éclairer plus précisément l'engagement des dirigeants de PME pour le label ENR. C'est en cela que l'ANT est un cadre théorique intégrateur, car il permet d'avoir deux représentations qui se nourrissent mutuellement, aussi bien collective qu'individuelle. Pour expliquer les raisons du succès relatif de la diffusion du label ENR au sein du réseau, nous proposons une typologie d'engagement responsable des dirigeants de PME. Le tableau 2 synthétise la méthode d'étude du déploiement du label ENR.

TAbleau 2. Méthode D’étude du déPloiement du label ENR

\begin{tabular}{ll}
\hline Déploiement du label & Description \\
\hline Analyse du contexte & $\begin{array}{l}\text { L'analyse du contexte est réalisée par les primum movens (D-F1), (CA- } \\
\text { 1) et (CA-2) } \\
\text { Trois constats découlent du diagnostic mené : le secteur du numérique } \\
\\
\text { est un secteur d'activité en cours de structuration (1), qui est marqué } \\
\text { par un déficit d'image dû à la bulle informatique (2). Les entreprises de } \\
\text { ce secteur sont en retard sur la question de la RSE (3) }\end{array}$ \\
\hline Identification & $\begin{array}{l}\text { Identification des actants humains : dirigeants de PME, membres des } \\
\text { clusters, animateurs des clusters, tiers certificateurs, experts... }\end{array}$ \\
problématisation & $\begin{array}{l}\text { Identifications des actants non humains : chartes éthiques existantes, } \\
\text { documents issus des groupes de travail... }\end{array}$ \\
& $\begin{array}{l}\text { La problématisation de la situation : comment intégrer des pratiques } \\
\text { responsables dans un secteur d'activité récent et dans lequel les } \\
\text { organisations n'ont pas, de prime abord, d'impact négatif majeur sur } \\
\text { l'environnement ? }\end{array}$ \\
\hline Traduction et point de & $\begin{array}{l}\text { Identification du traducteur : à la suite des primum movens, France IT } \\
\text { accepte d'endosser le rôle de traducteur }\end{array}$ \\
Eassage obligé & $\begin{array}{l}\text { Exemples de traduction : les concepteurs choisissent de développer } \\
\text { un label, dispositif apparaissant comme étant davantage à la portée } \\
\text { de dirigeants de TPE qu'une norme sectorielle ; France IT traduit } \\
\text { les intérêts de tous les actants en proposant une action collective de } \\
\text { formation sur le développement durable en partenariat avec le CIRIDD } \\
\text { Identification du point de passage obligé : création du label ENR }\end{array}$ \\
\hline
\end{tabular}




\begin{tabular}{ll}
\hline Déploiement du label & Description \\
\hline $\begin{array}{l}\text { Intéressement et } \\
\text { enrôlement }\end{array}$ & $\begin{array}{l}\text { Intéressement pour les acteurs : faire partie d'un collectif (France IT) } \\
\text { reconnu et légitimé au niveau national, bénéficier de l'expertise de } \\
\text { consultants RSE } \\
\text { Enrôlement des clusters et des entreprises adhérentes dans la } \\
\text { coconstruction du référentiel du label (retour d'expériences) }\end{array}$ \\
\hline $\begin{array}{l}\text { Mobilisation des porte- } \\
\text { parole }\end{array}$ & $\begin{array}{l}\text { Qui sont les porte-parole qui permettent au label de se diffuser ? Citons } \\
\text { notamment les instances de gouvernance des clusters, le CIRIDD, les } \\
\text { syndicats professionnels du comité d'attribution du label }\end{array}$ \\
\hline
\end{tabular}

\subsection{Proposition d'une typologie d'engagement pour le label ENR}

Dans le cadre de cette étude, nous avons décrit le processus de diffusion du label ENR au niveau organisationnel, France IT étant considérée comme une «méta-organisation » au sens de Amblard et al. (1996). Afin de comprendre les raisons de cette perte de vitesse, qui traduit in fine un manque d'alignement des traductions des différents acteurs, il est également pertinent de s'intéresser à la perception et aux motivations des dirigeants de PME vis-à-vis du dispositif et d'adopter ainsi une approche individuelle et complémentaire. Nous remarquons qu'au fil des vagues de labellisation, les profils des dirigeants évoluent en fonction de l'investissement et l'enrôlement de chacun autour de l'innovation.

Au cours de la première vague de labellisation, les entreprises labellisées sont très sensibles aux arguments du label relatifs à la réputation du secteur et au manque de prise en compte des enjeux sociétaux. Ainsi, deux primum movens à être aussi dirigeants de PME se sont enrôlés dans cette première vague, "et je me suis fait un point d'honneur d'être la première entreprise dans la première vague d'entreprises labellisées " (CA-2). Nous observons également des entreprises, dont les pratiques entrent en cohérence avec le label, «c'est vrai que le directeur de France IT nous a dit, c'est un label qui vous intéressera sûrement vis-à-vis de la philosophie de gestion qu'a l'entreprise. Le label ENR, il m'intéressa dès le départ »(CA-4).

Au cours de la deuxième vague de labellisation, les concepteurs du label peuvent compter sur des acteurs-cadres des clusters. Dans le cluster CB par exemple, c'est l'actuel président et dirigeant d'entreprise CB-5 qui s'engage dans un processus de labellisation.

" J'ai fait partie de la commission nationale pour France IT qui a mis en place le label ENR. [...] Donc forcément, dès que le cluster CB propose quelque chose, si on n'est pas au courant en amont parce que l'on travaille beaucoup avec le cluster $C B$, on est très attentif à ce qu'il propose."

Dans le cluster CE, le directeur (CE-D) s'est fixé des objectifs de labellisation annuelle : "J'essaye d'avoir deux entreprises du cluster labellisées par an. "

Le cas du dirigeant de CB-1 est également intéressant, car il justifie sa labellisation comme un moyen de montrer l'exemple aux autres membres du cluster depuis sa récente prise de fonction de président du cluster CB. 
La troisième vague se caractérise par la labellisation de deux entreprises du cluster CC (CC-1 et CC-2), qui vient de rejoindre l'association France IT, ainsi que la mobilisation importante d'entreprises (CB-2, CB-3, CB-4, CB-6) pour le cluster CB. Cet attrait pour le cluster CB s'explique par la mise en place d'un prédiagnostic de développement durable proposé gratuitement par le chargé de mission du cluster, et qui permet de mettre en lumière les axes d'amélioration des organisations, "nous nous efforçons d'intégrer des paramètres éthiques, sociaux et environnementaux dans notre organisation. Ce label était donc une bonne manière de nous situer par rapport aux efforts fournis » (CB-3).

Lors de la quatrième vague de labellisation, nous retrouvons des motivations communes aux précédentes à savoir des acteurs fortement impliqués dans leur cluster, une adéquation entre la philosophie managériale des dirigeants et le label. Néanmoins, nous observons l'expression de nouvelles formes d'intéressement vis-à-vis du label. Le dirigeant de CD-2 justifie sa motivation par une politique de labellisation globale de l'entreprise :

"On est de toute façon dans une démarche de label. Moi j’ai travaillé pas mal de temps en Allemagne, la labellisation fait partie de la vie de l'entreprise. Cela permet de tendre vers des bonnes pratiques et de formaliser les méthodes de travail. »

Le dirigeant de CG-1, quant à lui, est le premier à s'inscrire dans le processus de labellisation par réaction à la labellisation de concurrents, "on a des concurrents qui ont été labellisés, donc il serait bien que l'on candidate ». Ces différents positionnements de dirigeants de PME et leurs formes d'engagement RSE soulignent la particularité et l'intérêt d'observer le déploiement d'un label RSE dans un contexte PME. Contrairement à d'autres dispositifs de certification, la certification RSE implique d'impulser un dynamisme auprès des parties prenantes (Slager, Gond et Moon, 2012). Ce travail pour les traducteurs se voit complexifié dans un contexte de réseau de PME. Au gré des vagues de labellisation, nous observons plusieurs profils de dirigeants de PME. La nature de l'engagement RSE de ces derniers renvoie en partie à la typologie développée par Spence et Rutherfoord (2001), allant des dirigeants " convaincus » aux dirigeants qui instrumentalisent la RSE à des fins d'abord économiques. Le tableau 3 présente les types d'engagement des dirigeants pour le label ENR en fonction des mécanismes d'intéressement et d'enrôlement de France IT.

Tableau 3. Typologie D’engagement des dirigeants dans la labellisation

\begin{tabular}{ll}
\hline «Les précurseurs » & «Les ambassadeurs » \\
CA-2; CA-4 ; CD-1 & CB-5; CE-D ; CB-1 \\
Intéressement et enrôlement importants & Intéressement et enrôlement importants \\
Volonté de faire figure d'exemple et de & Mécanismes sociopolitiques \\
formaliser la stratégie RSE & Rôle de diffusion du label au sein des clusters \\
\hline «Les intéressés » & «Les opportunistes » \\
CB-2; CB-3 ; CB-4; CB-6 & CD-2; CG-1 \\
Intéressement important, enrôlement moindre & Intéressement important, enrôlement moindre \\
Intérêt pour le prédiagnostic de développement & Volonté, grâce au label, de contrer la \\
durable & concurrence \\
\hline
\end{tabular}


Dans une certaine mesure, le recueil des pratiques mentionnées par ces dirigeants d'entreprises nous permet de souligner la cohérence existante, entre leurs actions et leurs discours justifiant leur motivation pour la labellisation.

S'agissant des "précurseurs", les actions de ces acteurs sont toujours proactives même en postlabellisation. En témoigne d'ailleurs le rapprochement de CA-2 et CA-4 qui s'inscrivent aujourd'hui dans un partage de ressources et notamment de locaux dans le but de réduire leur consommation d'énergie, de partage de connaissances, tant celles relatives à leur cœur de métier que celles leur permettant d'ouvrir la focale de leur responsabilité sociétale respective.

Concernant les " ambassadeurs", nous retrouvons chez ces acteurs, en dehors de leurs pratiques organisationnelles propres, la volonté de diffuser le label. Ainsi au fil des vagues de labellisation, le nombre de candidatures dans le cluster CD par exemple reste constant.

Pour le groupe des «intéressés », il est pertinent de remarquer que l'importance du prédiagnostic RSE se retrouve dans les pratiques de ces organisations. Suite à l'obtention du label, ces entreprises se sont appuyées sur les axes d'amélioration mis en évidence par ce dispositif pour faire évoluer leurs pratiques. De la même manière, nous observons une diminution du nombre de candidatures pour la labellisation au sein du cluster CB du fait de l'arrêt du prédiagnostic.

Enfin, pour le groupe des « opportunistes", les organisations n'ont pas plus d'actions tournées vers les clients ou ayant des tendances plus commerciales que dans les autres entreprises labellisées. Elles ne s'inscrivent pas dans une stratégie de manipulation ou d'intégration symbolique de pratiques RSE, mais dans une stratégie réactive qui comprend une intégration concrète de pratiques RSE (Capron et Quairel-Lanoizelée, 2010).

Ces quatre types d'engagement témoignent de la crédibilité du label comme solution apportée au secteur. Toutefois, ce phénomène est marqué par le scepticisme de certains dirigeants vis-à-vis du label. Une partie de ce scepticisme s'explique par l'incapacité qu'ont certains dirigeants de PME à percevoir les opportunités des signaux externes (Torrès, 2003 ; 2008). Cette incapacité peut notamment être la résultante des effets de proxémie qui caractérise la PME et son dirigeant. Ainsi, ces acteurs se positionnent contre le dispositif de labellisation au regard de sa philosophie et comme solution apportée au secteur :

"Je vais poser une question toute bête, qu'est-ce que l'on entend par développement durable pour des gens qui font du logiciel? Je me pose la question en quoi une entreprise qui fait du logiciel peut modifier son comportement pour être plus verte, à part pour l'avoir sur le papier » (CN-A1).

Ces acteurs sont également critiques vis-à-vis du manque de visibilité et de crédibilité du label (CN-B2), ou plus largement de la forme du dispositif (CN-A2).

Il est alors possible d'avancer que ces différents profils d'engagement responsable - degré d'engagement fort versus degré d'engagement faible - et les attentes qui en découlent n'ont pas été suffisamment étudiés par les primum movens, mais surtout par le traducteur au moment de l'étape de problématisation. Cela aurait notamment permis d'identifier et de proposer des éléments matériels ou cognitifs qui auraient facilité l'intéressement des dirigeants de PME et de ce fait leur enrôlement dans la diffusion de l'innovation. 


\section{DISCUSSION ET CONCLUSION}

L’objectif de ce travail réside dans l'analyse de la diffusion d'un label RSE au sein d'un réseau de clusters de PME. Nous nous sommes ainsi focalisés sur la diffusion du label Entreprise Numérique Responsable au sein de France IT pour montrer comment les faits se sont construits à travers un processus de traduction.

Sur le plan théorique, notre démarche s'inscrit autour de plusieurs postulats. Les outils relatifs à la RSE et notamment les dispositifs de certification sont de plus en plus nombreux (Capron et Quairel-Lanoizelée, 2010), mais seul un nombre restreint d'outils sont appropriés aux PME (Perrini et Tencati, 2006). Aussi, il apparaît pertinent d'observer le déploiement d'un dispositif de labellisation RSE spécifique pour ce type d'organisations. Notons également l'importance du déploiement d'un tel dispositif dans un contexte de clusters, cet environnement devant faciliter la diffusion d'un projet de cette nature. Afin de comprendre comment un objet spécifique, comme un label RSE conçu pour les PME, peut être diffusé et intégré au sein d'un réseau de clusters, nous avons mobilisé l'approche théorique de l'acteurréseau (Akrich, Callon et Latour, 1988) qui permet d'appréhender la diffusion de l'innovation RSE par les acteurs humains et non humains au sein du réseau. Trois étapes majeures ont tout d'abord été identifiées, favorisant la diffusion et la pérennisation du label. Puis, nous avons mis en évidence une typologie d'engagement en faveur du label selon les mécanismes « d'intéressement » et d'enrôlement identifiés au sein du réseau.

Cette démarche de nature exploratoire a permis de montrer l'intérêt opératoire de la théorie de l'acteur-réseau dans l'élaboration d'un outil innovant de RSE. Ce travail s'inscrit dans la littérature existante sur l'engagement RSE en PME (Spence et Rutherfoord, 2001 ; Rooke et Torbert, 2005 ; Boiral, Cayer et Baron, 2009 ; Boiral, Baron et Gunnlaugsin, 2014).

Nous montrons ici tout l'intérêt d'articuler les dimensions collective et individuelle dans l'analyse, en rapprochant le travail de traduction aux mécanismes d'engagement de la RSE par les dirigeants de PME. Ce travail souligne également l'importance de mobiliser dès la phase amont l'ensemble des acteurs du réseau (humains et non-humains) pour favoriser cette diffusion de l'innovation. Ceci est d'autant plus vrai dans le contexte d'un dispositif de certification RSE. Comme l'expliquent Slager, Gond et Moon (2012), un dispositif RSE de par sa nature implique une innovation continue du fait du dynamisme des enjeux à prendre en considération et des évolutions des parties prenantes (Christmann et Taylor, 2006). Aussi, il semble indispensable pour de tels dispositifs de s'ouvrir régulièrement à de nouveaux acteurs, ces derniers étant capables d'apporter un éclairage nouveau sur la question des parties prenantes et des enjeux dans une logique de boucles d'innovation constantes.

Les éléments présentés dans les résultats confirment le développement de notre cadre théorique concernant l'intérêt de diffuser un dispositif RSE au sein d'un réseau de clusters (Chalaye et Massard, 2009). En effet, les premières étapes du processus de diffusion mettent en évidence le réseau de clusters comme un appui dans la diffusion du label RSE. Ainsi les concepteurs s'appuient sur les outils préexistants, à la fois comme base de diagnostic et d'élaboration du nouveau dispositif. Les démarches collectives existantes leur donnent également une capacité de mobilisation et d'implication des acteurs plus importante. Cependant, si l'identification d'un primum movens et d'un traducteur est essentielle à la compréhension d'une situation de changement (Walsh et Renaud, 2010), elle n'est pas suffisante. Il faut 
également prendre en considération les attentes de tous les acteurs impliqués dans le réseau, en les convainquant à prendre des responsabilités au sein du réseau, favorisant ainsi la diffusion de l'innovation. Toute la difficulté réside dans l'identification du degré d'engagement en faveur du dispositif de RSE. Repérer dès la phase amont du projet les attentes et besoins de chaque acteur permet ensuite de lui proposer, compte tenu de son degré d'engagement responsable - des incitations aussi bien matérielles que cognitives adaptées à son profil, ce qui favorise son enrôlement dans le réseau et sa participation à la diffusion de l'innovation.

À l'inverse, la diffusion d'un dispositif de certification RSE destiné aux PME nous amène à observer un résultat contre-intuitif. La littérature scientifique met en évidence le fait que peu d'outils RSE sont conçus pour les PME (Perrini et Tencati, 2006). D’un point de vue empirique, les concepteurs du dispositif étudiés font un constat similaire. Aussi, le développement d'un dispositif de labellisation RSE destiné aux PME devrait voir sa diffusion facilitée par l'adéquation avec le type d'organisations cibles. Or, nous remarquons, dans le cas étudié, que le dispositif de certification RSE, de par son objet, implique des natures d'engagement diverses de la part des dirigeants de PME. En effet, la labellisation RSE interroge à la fois les entreprises dans leurs fondements, dans leurs relations avec leur environnement, mais aussi dans le calcul qui est fait entre objectifs économiques et engagement environnemental et sociétal (Courrent, 2012 ; Spence et Rutherfoord, 2001). Même si la diffusion d'un dispositif de certification RSE au sein d'un réseau de PME n'est pas impossible, elle est rendue bien plus complexe comme le montre ce cas d'étude. En effet, les différents profils d'engagement de la part des dirigeants de PME accroissent la complexité pour les primum movens, de trouver des terrains d'entente (Akrich et al., 1988) afin de concilier des logiques qui apparaissent comme très éloignées, et en particulier auprès des dirigeants de PME qui ne perçoivent aucun intérêt éthique ou commercial.

Malgré cela, cette étude de la diffusion de la RSE au sein de PME, par le biais d'un dispositif de labellisation, s'inscrit dans un secteur qui de par sa nature offre autant de réponses que de questions aux enjeux sociétaux posés aux entreprises. En effet, si des acteurs du secteur ont fait le choix de développer un label RSE sectoriel au travers de France IT, c'est précisément parce qu'un certain nombre de pratiques inhérentes aux entreprises du numérique ne sont pas neutres pour leur environnement (Sama et Shoaf, 2002). Au travers de la première étape de formation du réseau, plusieurs problématiques ont été mises en évidence. Cellesci peuvent être sociales, comme le niveau d'investissement de collaborateurs dans les projets des entreprises au détriment de leur santé. Elles peuvent être relatives à la loyauté des pratiques, que ce soit vis-à-vis des concurrents et des clients, ou encore environnementales quand elles sont liées à la consommation d'énergie des datacenters.

Si l'objet du dispositif de certification rend sa diffusion complexe dans un contexte PME, nous observons également les limites de la forme de ce dispositif. En effet, en développant un dispositif de certification sous la forme d'un label, les concepteurs se posent plusieurs limites qui viennent contraindre la capacité d'innovation du dispositif et l'appropriation qui peut en être faite par les dirigeants de PME. Comme nous le remarquons dans la présentation des résultats, le label RSE se doit d'être relativement indépendant de ses créateurs, une fois diffusé, afin d'assurer la crédibilité de celui-ci et le niveau de rigueur de l'audit qui en découle. Aussi, les évolutions, les souhaits que pourraient exprimer les dirigeants de PME relatifs aux questions liées à la dynamique des enjeux, des parties prenantes, ainsi qu'à la nature de l'engagement de ces dirigeants ne peuvent être pleinement prises en compte au 
point d'influencer le dispositif. Cette impasse est contraire à ce que Slager, Gond et Moon (2012) démontrent. En effet, la diffusion réussie d'un tel dispositif RSE s'accompagne de la possibilité de faire évoluer ledit dispositif, en intégrant de nouveaux acteurs et les enjeux qu'ils portent dans une succession de boucles d'innovation continue.

Sur le plan managérial, ce travail souligne l'intérêt pour des réseaux de la coconstruction de l'innovation de manière collective. La diffusion de l'innovation ne doit pas être considérée comme un processus linéaire, mais bien comme une coconstruction de la part des dirigeants qui sont engagés dans la diffusion du label. Les concepteurs du label disposent ainsi d'une grille leur permettant d'anticiper la réception du dispositif par les dirigeants, les facteurs de motivation, les freins et les attitudes de ces derniers dans le cadre de la diffusion de l'innovation. Il est ainsi envisageable de construire le travail de traduction soit en s'appuyant sur certains profils de dirigeants pour faciliter la diffusion du dispositif, par le biais des " ambassadeurs " par exemple ; soit en prenant soin d'intégrer les mécanismes d'engagement au dispositif, comme la question de la visibilité de celui-ci, critère important pour les « opportunistes".

Ces différents apports doivent toutefois être considérés au regard des limites de cette recherche qui représentent autant de voies de recherche futures. En premier lieu, il est important de souligner le caractère exploratoire de ce travail. En observant un dispositif de labellisation dans un contexte de réseaux de clusters, cette recherche s'inscrit dans un cadre à la fois riche et particulier sur le plan empirique. Le caractère longitudinal de celle-ci offre à l'issue de trois années d'observation les enseignements susmentionnés. Cela dit, cet aspect longitudinal représente aussi des principales limites de l'étude. Si les étapes de traduction présentées ne vont plus évoluer, la nature de l'engagement des dirigeants peut encore se modifier, tant que le phénomène est en cours. Il est envisagé de poursuivre les entretiens avec les participants pour analyser le degré d'appropriation des principes de RSE diffusés par le label, tant au niveau individuel qu'organisationnel. Pour mener ces futures recherches, la méthodologie de la cartographie cognitive est pressentie (Cossette et Audet, 1994).

\section{RÉFÉRENCES}

Ajzen, M., Rondeaux, G., Pichault, F. et Taskin, L. (2016). Performance et innovation en PME : une relation à questionner. Revue internationale PME, 29(2), 65-94.

Akrich, M., Callon, M. et Latour, B. (1988). À quoi tient le succès des innovations, deuxième épisode : l'art de choisir les bons porte-paroles. Annales des Mines, 12, 14-29.

Akrich, M., Callon, M. et Latour, B. (2006). Sociologie de la traduction : textes fondateurs. Paris, École des mines de Paris.

Aldrich, H.E. et Fiol, C.M. (1994). Fools rush in ? The institutional context of industry creation. Academy of Management Review, 19(4), 645-670.

Amblard, H., Bernoux, P., Herreros, G. et Livian, Y.-F. (1996). Les Nouvelles Approches sociologiques des organisations ( $3^{\mathrm{e}}$ édition). Paris, Éditions du Seuil.

Barham, E. (2002). Towards a theory of values-based labeling. Agriculture and Human Values, 19(4), 349-360. 
BARZI, R. (2011). PME et agilité organisationnelle : une étude exploratoire. Innovations, 2(35), 29-45.

Berger-Douce, S. (2008). Rentabilité et pratiques de RSE en milieu PME-Premiers résultats d'une étude française. Revue management \& avenir, 1(15), 9-29.

Bernard, R.H. et Ryan, G.W. (2010). Analyzing qualitative data. Systematic approaches. Thousand Oaks, Sage Publications.

BLOoR, D. (1976). Knowledge and social imagery. Chicago, University of Chicago Press.

Boiral, O., Baron, C. et Gunnlaugsin, O. (2014). Environmental leadership and consciousness : a case study among canadian SMEs. Journal of Business Ethics, 123(3), 363-383.

Boiral, O., CAYer, M. et BAron, C. (2009). The action logics of environmental leadership : a developmental perspective. Journal of Business Ethics, 85(4), 479-499.

Bonneveux, E. et Calmé, I. (2015). La construction du réseau autour d'une démarche innovante de RSE : les apports de la sociologie de la traduction. Revue Interdisciplinaire Management, Homme(s) \& Entreprise, 16, 18-36.

Bonneveux, E., Calmé, I. et Soparnot, R. (2011). La diffusion d'une démarche RSE innovante au sein d'un réseau de PME : le cas du Centre des jeunes dirigeants. Revue Gestion 2000, 28(2), 69-90.

Bou SABA, M. (2011). L'implantation d'un outil d'intelligence collective. Un essai d'observation et d'interprétation. L'outil COOPERFIC pour les coopératives agricoles du Languedoc-Roussillon (thèse de doctorat en sciences de gestion). Montpellier, Université de Montpellier 2.

Brundtland, G.H. (1987). Our common future (World Commission on Environment and Development). Oxford Paperbacks, Oxford University Press.

Cajaiba-santana, G. (2014). Social innovation : moving the field forward. A conceptual framework. Technological Forecasting \& Social Change, 82, 42-51.

CAllon, M. (1986). Éléments pour une sociologie de la traduction, la domestication des coquilles Saint-Jacques et des marins pêcheurs de la baie de Saint-Brieuc. L’Année Sociologique, 36, 169-208.

Callon, M. (1988). La science et ses réseaux. Genèse et circulation des faits scientifiques. Paris, La Découverte.

CAllon, M. (2006). Les réseaux sociaux à l'aune de la théorie de l'acteur-réseau. Sociologies Pratiques, $13,37-44$.

Callon, M. et Latour, B. (1991). La science telle qu'elle se fait. Anthologie de la sociologie des sciences de langue anglaise. Paris, La Découverte.

Callon, M., Lascoumes, P. et Barthe, Y. (2001). Agir dans un monde incertain. Essai sur la démocratie technique. Paris, Éditions du Seuil.

Capron, M. et Quairel-Lanoizelée, F. (2010). La responsabilité sociale de l'entreprise. Paris, La Découverte.

Carpenter, M. et Larceneux, F. (2008). Label equity and the effectiveness of values-based labels : an experiment with two French protected geographic indication labels. International Journal of Consumer Studies, 32(5), 499-507.

CE (2013). Guide to social innovation. Bruxelles, Commission européenne.

Chalaye, S. et Massard, N. (2009). Les Clusters: diversité des pratiques et mesures de performance. Revue d'économie industrielle, 128, 153-176. 
Christmann, P. et Taylor, G. (2006). Firm self-regulation through international certifiable standards : determinants of symbolic versus substantive implementation. Journal of International Business Studies, 37(6), 863-878.

Cossette, P. et Audet, M. (1994). Qu'est-ce qu'une carte cognitive ? Dans Cartes cognitives et organisation (p. 13-34). Presses de l'Université Laval/Éditions Eska.

Courrent, J.-M. (2012). RSE et développement durable en PME. Comprendre pour agir. Bruxelles, De Boeck.

De George, R.T. (2003). The Ethics of information technology and business. Malden, Blackwell Publishing.

Depret, M.H. et Hamdouch, A. (2010). Politiques d'innovation, espace régional et dynamique des territoires : un essai de caractérisation dans le contexte français. Innovations, 3(33), 85-104.

Devaujany, F.-X. (2006). Pour une théorie de l'appropriation des outils de gestion : vers un dépassement de l'opposition conception-usage. Revue management \& avenir, 3(9), 109-126.

DiMaggio, P. et Powell, W. (1983). The Iron cage revisited : institutional isomorphism and collective rationality in organizational fields. American Sociological Review, 48, 147-160.

Fieseler, C., Bucher, E. et Hoffmann, C.P. (2017). Unfairness by design ? The perceived fairness of digital labor on crowdworking platforms. Journal of Business Ethics. Récupéré le $1^{\text {er }}$ mai 2019 sur le site : https://link.springer.com/article/10.1007\%2Fs10551-017-3607-2.

Gautier, A., Badea, A. et Berger-Douce, S. (2017). Les PME : un contexte pertinent pour l'étude des leviers de la création de valeur partagée. Management international, 21(4), 109-125.

Greimas, A.J. (1986). Sémantique structurale. Paris, Presses universitaires de France.

Jenkins, H. (2006). Small business champions for corporate social responsibility. Journal of Business Ethics, 67(3), 241-256.

Koenn, D. (2003). The nature of and conditions for online trust. Journal of Business Ethics, 43, 3-19.

LAPointe, A. et Gendron, C. (2005). La responsabilité sociale dans la PME : une option marginale ou enjeu vital ? Les Cahiers de la Chaire, (6). Montréal, UQAM.

Larceneux, F. (2003). Segmentation des signes de qualité : labels expérientiels et labels techniques. Décisions Marketing, 29, 35-46.

Latour, B. (1992). Ces réseaux que la raison ignore. Paris, Éditions L’Harmattan.

Latour, B. et Lemonnier, P. (1994). De la préhistoire aux missiles balistiques : l'intelligence sociale des techniques. Paris, La Découverte.

Lemaire, C. (2013). Le processus de construction d'un outil de contrôle de gestion interorganisationnel. Le cas de l'expérimentation d'un outil de pilotage de la performance dans le secteur médico-social (thèse de doctorat en sciences de gestion). Strasbourg, Université de Strasbourg.

Mahé de Boislandelle, H. (1996). L’effet de grossissement chez le dirigeant de PME : ses incidences sur le plan du management des hommes et de la GRH. $3^{e}$ Congrès international francophone PME. Trois-Rivières, Québec, 25-27 juin.

McEACHern, M.G. (2008). Guest editorial : the consumer and values-based labels. International Journal of Consumer Studies, 32(5), 405-406. 
McEachern, M.G. et Warnaby, G. (2008). Exploring the relationship between consumer knowledge and purchase behaviour of value-based labels. International Journal of Consumer Studies, 32(5), 414-426.

Moisdon, J.-C. (1997). Du mode d'existence des outils de gestion, les instruments de gestion à l'épreuve de l'organisation. Paris, Seli Arslan.

Murillo, D. et Lozano, J. (2006). SMEs and CSR : an approach to CSR in their own words. Journal of Business Ethics, 67(3), 227-240.

Nobre, T. et Zawadzki, C. (2015). Analyse par la théorie de la traduction de l'abandon et du détournement d'outils lors de l'introduction d'un contrôle de gestion en PME. Finance Contrôle Stratégie, $18(1), 1-20$.

Nwagwu, W.E. et IBeKu, S. (2016). Understanding the innovativeness of information technology products and service providers in an IT cluster in Nigeria. SA Jnl Libs \& Info Sci, 82(1), 36-52.

ObServatoire du numérique (2015). Chiffres clés du numérique. Récupéré le 20 novembre 2016 du site : http://www.observatoire-du-numerique.fr/.

O'Rourke, D. (2006). Multi-stakeholder regulation : privatizing or socializing global labor standards? World Development, 34(5), 899-918.

Oueghlissi, R. (2013). La RSE et les PME. Analyse descriptive à partir de l'enquête COI 2006. Revue française de gestion, 7(236), 163-180.

Panteli, A., Stack, J. et Ramsay, H. (1999). Ethics in the IT industry. Journal of Business Ethics, 22, 51-61.

Pasquero, J, (2004). Responsabilité sociale de l'entreprise : les approches nord-américaine. Dans J. Igalens (dir.), Tous responsables. Paris, Éditions d'Organisation.

Perrini, F. (2006). SMEs and CSR theory : evidence and implications from an italian perspective. Journal of Business Ethics, 67(3), 305-316.

Perrini, F. et Tencati, A. (2006). Sustainability and stakeholder management : the need for new corporate performance evaluation and reporting systems. Business strategy and the environment, 15(5), 296-308.

Porter, M.E. et Kramer, M.R. (2011). The big idea, creating shared value. Harvard Business Review, $89(1-2), 62-77$.

QuAirel, F. (2006). Contrôle de la performance globale et RSE. $27^{\circ}$ Congrès de l'AFC. Comptabilité, Contrôle, Audit et Institution(s). Tunis, Tunisie, 10-12 mai.

Robert-Demontrond, P. et Joyeau, A. (2007). Le label commerce équitable comme praxème : diversité des acteurs, diversification des significations. Revue de l'organisation responsable, 2(2), 54-69.

Rooke, D. et Torbert, N. (2005). Seven transformation of leadership. Harvard Business Review, 83(4), 66-76.

Sama, L.M. et Shoaf, V. (2002). Ethics on the Web : applying moral decision-making to the new media. Journal of Business Ethics, 36, 93-103.

Shilton, K. et Greene, D. (2017). Linking platforms, practices, and developer ethics : levers for privacy discourse in mobile application development. Journal of Business Ethics. Récupéré le $1^{\text {er }}$ mai 2019 sur le site : https://link.springer.com/article/10.1007/s10551-017-3504-8.

SкоG, D.A. (2016). Local game, global rules : exploring technological heterogeneity exploitation in digital creative cluster evolution. Industry and Innovation, 23(6), 531-550. 
Slager, R., Gond, J.-P. et Moon, J. (2012). Standardization as institutional work : the regulatory power of a responsible investment standard. Organization Studies, 33(5-6), 763-790.

SPenCE, L.J. et Rutherfoord, R. (2001). Social responsibility, profit maximisation and the small firm owner-manager. Journal of Small Business and Enterprise Development, 8(2), 126-139.

TANguy, L. (2011). De la théorie des coûts de transaction à une économie des coûts de traduction: l'émergence d'un centre de services mutualisés comme dispositif de contrôle interorganisationnel (thèse de doctorat en sciences de gestion). Paris, HEC Paris.

Torrès, O. (2003). Petitesse des entreprises et grossissement des effets de proximité. Revue française de gestion, 3(144), 119-138.

Torrès, O. (2008). L’ambivalence du management de proximité des TPE. Dans A. Jaouen et O. Torrès (dir.), Les Très Petites Entreprises : un management de proximité. Paris, Hermès.

Trépanier, M. et AkA, K.G. (2017). L'analyse des réseaux dans les activités d'innovation des PME : le rôle oublié de l'homophilie. Revue internationale PME, 30(2), 33-60.

VAN DER Yeught, C. et Bon, V. (2016). Quand une innovation sociale produit de l'innovation responsable. Revue française de gestion, 42(255), 27-40.

Walsh, I. et Renaud, A. (2010). La théorie de la traduction revisitée ou la conduite du changement traduit. Application à un cas de fusion-acquisition nécessitant un changement de système d'information. Revue management \& avenir, 9(39), 283-302.

Wymenga, P., Spanikova, V., Barker, A., Konings, J. et Canton, E. (2012). EU SMEs in 2012 : at the crossroads. Annual report on small and medium-sized enterprises in the EU.

YIN, R.K. (2014). Case study research : design and methods (5 édition). Thousand Oaks, Sage Publications. 